Presented review of literature data (medline, eLIBRARY.RU, PabMed) identifies typical problems of parents whose children were born with cleft lip and palate (RGN), as well as research directions in improving the organization of medical care for parents and patients with UAH in solving these problems. Analysis of domestic and foreign authors on MEDLINE databases, eLIBRARY.RU, PabMed Still lacks research on the experiences of parents with RSN, what typical problems they encountered in the maternity hospital and, especially, at home. It is now necessary to carry out research that has traditionally been rely not only on psychological approaches, but also on broader prospects for research in the field sociology, social policy, nursing and health care using both qualitative and quantitative methods. In addition, there is a lack of research in the field of cleft lip and palate to study the experience and needs of parents at different stages of their children's lives.

\section{WILLIAMS-BEUREN SYNDROME: A CASE REPORTS}

Dorian Laslo*, Višnja Tomac, Silvija Pušeljić. Faculty of medicine Osijek, Josip Juraj Strossmayer University of Osijek

\subsection{6/archdischild-2021-europaediatrics.93}

Introduction Williams-Beuren syndrome (WBS) is developmental disorder caused by microdeletion of genes from chromosome 7. It is estimated in 1 of 10000 people. WBS could be autosomal-dominant inherited, but usually, it is caused by de novo microdeletion of 26-28 genes on chromosome 7 . Usually genes such as CLIP2, NCF1, ELN, GTF2I, GTF2IRD1 and LIMK1 are deleted. ELN gene is detected as a cause of supravalvular aortic stenosis, while absence of NCF1 gene is related to hypertension. Patients with WBS are characterized by cardiovascular diseases, facial dysmorphic features, intellectual disability, unique personality character and endocrine abnormalities.

Case Report We present the case of three patients, two girls at the ages of one and three years and a boy at the age of two years. All three patients have healthy parents and brothers and sisters without known chronic or genetic diseases. All three were presented with facial dysmorphic features which include broad nasal bridge, microretrognathia, large mouth, upper lip is thin while lower lip is thicker. All three patients are cognitively deficient and they have speech problems. The female patient at the age of three years also has hypertension, small teeth, larger neurocranium, atrial and ventricular (muscular) septal defect, gastroesophageal reflux disease (GERD), hypothonia and endocrine disorders; hypercalcemia, hypercalciuria, hypothyroidism and early puberty. The male patient also has prominent ears, palmar crease on left palm, pulmonary stenosis with mitral regurgitation and bilateral inguinal hernia. All three patients were suspective for WBS.

Karyograms of all patients were normal, but FISH analysis performed with ELN primer for detection of WBS proved microdeletion on chromosome 7 in region 11 (7q11).

Conclusion WBS is rare disorder characterised by wide range of symptoms and signs.

Clinical diagnostic criteria are available for WBS, but conformation of diagnosis requires detection of microdeletion on chromosome $7 \mathrm{q} 11.2$. Early recognition of specific patterns of the disorder, such as facial dysmorphic features together with heart abnormalities, cognitive deficiency and vision problems, is crucial because those patients need to take speech therapist and psychologist therapy as soon as possible because it improves their integration in social environment. Management is focused on treatment of symptoms (eg. hypertension, hypercalcemia), psychological and psychiatric evaluation and speech therapy.

\section{CLINICAL AND GENETIC SPECTRUM OF DYSTROGLYCANOPATHY DUE TO POMGNT1 MUTATIONS IN RUSSIAN PATIENTS}

OB Kondakova*, KV Savostyanov, KA Kazakova, AA Pushkov, AA Lyalina, YI Davidova, OS Kuprianova, DI Grebenkin. National Medical Research Center for Children's Health

\subsection{6/archdischild-2021-europaediatrics.94}

Dystroglycanopathies are the heterogeneous group of hereditary disorders, caused by the abnormal glycosylation of $\alpha$-dystroglycan. The most common dystroglycanopathy is muscleeye-brain disease (MEB) associated with mutations in the POMGNT1 gene. MEB is autosomal recessive disease characterized by congenital muscular dystrophy, ocular abnormalities and brain malformation. The goal of the study is an analysis of clinical findings, laboratory features and results of instrumental researches.

Molecular genetic diagnostics was performed using full exome sequencing. All patients and all parents were confirmed by Sanger sequening.

We observed 3 boys with MEB disease aged from 25 to 118 months, averaging about 7 years (83 months). The one patient had exceed average values of height and weight at birth, two the other children had normal ranges. All children had severe motor development delay, only one patient could walked without support. Two older patients had mental retardation and lack of speech development. Language skills represented as vocalizations. Physical growth of our patients fluctuated from 3 to 75 percentiles for height and weight. The other clinical features include hypotonia and strabismus (all patients), autistic behavior (2 patients), ataxia, seizures, hepatomegaly (one in each patient). Dysmorphic features were non-specific.

Ophthalmological examination revealed congenital high myopia (2 patients), partial atrophy of an optic nerve (2 patient), nystagmus (1 patient), astigmatism (1 patient), retinal atrophy (1 patient).

Biochemical analysis showed elevated creatine kinase from 1874 to 6266 (averaging $3754 \mathrm{U} / \mathrm{L})$, ALT from 42 to $93(68$ $\mathrm{U} / \mathrm{L})$, AST from 53 to $106(80 \mathrm{U} / \mathrm{L}), \mathrm{LDH}$ from 370 to 503 (437 U/L).

Electromyographic examination has showed that all children had signs of primary muscle lesion. Muscle MRI has displayed a severe atrophy muscles and fatty infiltration in one patient. MRI findings have reported pachygyria and ventriculomegaly (all patients), hypoplasia cerebellum, corpus callosum, pons (2 patients), hypoplasia of temporal lobes (1 patients), cerebellum cysts (2 patients). EEG did not reveal epileptic form activity.

We revealed compound heterozygous mutations in all three patients. These were five different mutations: missense c.385C > T (p.R129W) and c.1325G >A (p.R442H), nonsense 